\title{
Multi-Source Spatial Entity Linkage
}

\author{
Suela Isaj \\ Aalborg University \\ suela@cs.aau.dk
}

\author{
Esteban Zimányi \\ Université libre de Bruxelles \\ ezimanyi@ulb.ac.be
}

\author{
Torben Bach Pedersen \\ Aalborg University \\ tbp@cs.aau.dk
}

\begin{abstract}
Besides the traditional cartographic data sources, spatial information can also be derived from location-based sources. Location-based sources offer rich spatial information describing the semantics of locations. However, even though different location-based sources refer to the same physical world, each one has only partial coverage of the spatial entities of interest, describe them with different attributes, and sometimes provide contradicting information. Hence, the problem of finding which pairs of spatial entities belong to the same physical spatial entity demands specific attention. We propose a solution (QuadSky) to the problem of spatial entity linkage across diverse location-based sources. QuadSky starts with a spatial blocking technique (QuadFlex) that inherits the concept and the complexity from the quadtree algorithm but improves the splitting technique not to separate nearby points. After comparing the spatial entities of the same block, we propose a novel algorithm, referred to as $S k y E x$ that separates the pairs considered as a match (positive class) from the rest (negative class) by using Pareto optimality. SkyEx does not require weights on the attributes, scoring function, or a training set. QuadSky achieves 0.85 precision and 0.85 recall for a manually labeled dataset of 1,500 pairs and 0.87 precision and 0.6 recall for a semi-manually labeled dataset of 777,452 pairs. Moreover, QuadSky provides the best trade-off between precision and recall and consequently, the best $F$-measure compared to the existing baselines.
\end{abstract}

\section{CCS CONCEPTS}

- Information systems $\rightarrow$ Entity resolution; Geographic information systems.

\section{INTRODUCTION}

Web data and social networks are growing in terms of information volume and heterogeneity. Almost all online sources offer the possibility to introduce locations (geo-tagged entities accompanied by semantic details). A specific type of sources is location-based sources, whose primary focus is locations. In contrast to cartographic data sources, locations in location-based sources have a hybrid form that stands between a spatial object and an entity. We refer to them as spatial entities since they are spatially located but also identified by several other attributes such as the name of the location, the address, the phone, keywords, etc. Given that spatial entities provide

Permission to make digital or hard copies of all or part of this work for personal or classroom use is granted without fee provided that copies are not made or distributed for profit or commercial advantage and that copies bear this notice and the full citation on the first page. Copyrights for components of this work owned by others than the author(s) must be honored. Abstracting with credit is permitted. To copy otherwise, or republish, to post on servers or to redistribute to lists, requires prior specific permission and/or a fee. Request permissions from permissions@acm.org.

SSTD '19, August 19-21, 2019, Vienna, Austria

(c) 2019 Copyright held by the owner/author(s). Publication rights licensed to ACM. ACM ISBN 978-1-4503-6280-1/19/08 . \$ \$15.00

https://doi.org/10.1145/3340964.3340979 richer semantics, several systems that rely on spatial information such as geo-recommender systems, selecting influential locations, search engines that use geo-preferences, etc. could improve further their results by using spatial entities instead of spatial objects. Hence, it is of interest to collect and integrate spatial entities from location-based sources.

While a spatial object is identified only by the coordinates, this is not the case for spatial entities. Different spatial entities might co-exist in the same coordinates (shops in a shopping mall), or the same entity might be located in different but nearby coordinates across different sources. The identity of a spatial entity is the combination of several attributes. Nevertheless, the identity of a spatial entity is sometimes difficult to infer due to the inconsistencies in the sources; each location-based source contains different attributes; some attributes might be missing and even contradicting. For example, the spatial entity "Lygten" is located in (57.436 10.534) and associated to keywords such as "coffee", "tea", and "cocoa and spices" in source A. In the meantime, source B contains the spatial entity named "Restaurant Lygten" in (57.435 10.533), described by the keyword "restaurant". We need a technique that can automatically decide whether these two spatial entities are the same real-world entity. The problem of finding which spatial entities belong to the same physical entity is referred to as spatial entity linkage or spatial entity resolution. According to [5], entity linkage establishes a link between spatial entities, whereas entity resolution goes further by merging related entities into one merged representative entity. Since we do not perform the latter, we use the term entity linkage.

There are several works that apply entity linkage in various fields $[9,10,12,12,13,15,20,20,22,31,35]$ but little regarding spatial entities $[4,16,21]$. In general, the entities studied in the current entity linkage paper refer to people; thus, the methodologies and the models are based on the similarities that two records of the same individual would reveal. Moreover, these works discard the spatial character of spatial entities. As for the works in spatial entity integration $[4,16,21]$, their main contribution is a tool rather than an algorithm. What is more, the methods propose arbitrarily attribute weights and score functions without experimentation nor evaluation. To sum up, with the growing amount of information from location-based sources and the necessity for richer spatial information, a method to link spatial entities across different sources is needed.

In this paper, we address the problem of spatial entity linkage across different location-based sources. First, we propose a method that uses the geo-coordinates to arrange the spatial entities into blocks. Then, we pairwise compare the attributes of the spatial entities. Finally, we introduce a novel technique for deciding whether the pairs of compared entities belong to the same physical entity. Our contributions are:

(1) We introduce QuadSky, a technique for linking spatial entities and we evaluate it on real-world data from four locationbased sources. 
(2) We propose an algorithm called QuadFlex that organizes the spatial entities into blocks based on their spatial proximity. QuadFlex inherits the concept and the complexity of quadtrees but avoids assigning nearby points in different blocks.

(3) To decide whether a of pair spatial entities refers to the same physical entity or not, we propose a flexible technique $(S k y E x)$ that is based on the concept of Pareto optimality and needs no weights, no scoring functions, nor a training set.

The remainder of the paper is structured as follows: first, we describe and compare to state of the art in Section 2; then, we detail our proposed algorithm in Section 3; later, we provide experiments regarding the performance of our proposed solution in Section 4 and finally, we conclude in Section 5.

\section{RELATED WORK}

The spatial entity linkage problem has not been explicitly addressed, but there are several works on entity resolution and also on spatial data integration. The general data integration problem covers three aspects: schema matching, entity resolution and data fusion $[8,12]$. In general, there are various works that tackle the entity resolution problem but few that deal with spatial entities.

Entity resolution. The entity resolution problem has been referred in the literature with multiple terms including deduplication, entity linkage, and entity matching. Entity resolution has been used in various fields such as matching profiles in social networks [31], bioinformatics data [35], biomedical data [7], publication data $[12,20]$, genealogical data [10], product data $[12,20]$, etc. The attributes of the entities are compared, and a similarity value is assigned. The decision of whether to link two entities or not is usually based on a scoring function. However, finding an appropriate similarity function that combines the similarities of attributes and decides on whether to link or not the entities is often difficult. Several works use a training set to learn a classifier [9, 13, 25], others base the decision on a threshold derived through experiments $[22,26]$. Other approaches that deal with uncertainty are described in the survey of Magnani and Montesi [19], including probabilistic, rule-based probabilistic, fuzzy, and preference-based relationships between records as well as aggregation of multi-matches. Finally, the decision on whether to link to entities or not can also be based on the feedback of an oracle $[12,20]$ or of a user [20].

Spatial data integration. There are several works on integrating geographical data; some integrate purely spatial objects, some spatial entities. Spatial objects differ from spatial entities mainly because a spatial object is fully determined by the coordinates and the spatial shape whereas a spatial entity, in addition to being geolocated, has a well-defined identity (name, phone, opening hours, categories). The works on spatial object integration aim to create a unified spatial representation of the spatial objects that come from single/multiple sources. The solutions in [1, 2, 32, 33] are purely spatial and discuss the integration of spatial objects originating from sensors and radars to have a better representation of the surface in $2 \mathrm{D}$ or even in 3D. Road network integration is tackled by Schafers at al [29] where rules are used to detect matching and non-matching roads. The matching is performed on the similarity of the roads in terms of the length, angles, shape, as well as the name of the street if available. Nonetheless, this approach is based on roads only and cannot apply to the linking of spatial entities.

Entity resolution of spatial entities has been discussed in $[4,16$, $21,30]$. The work in [30] is a bridge between the works in spatial data integration and spatial entity linkage because the entities have names, coordinates, and types but similarly to spatial objects, they refer to landscapes (rivers, deserts, mountains, etc.). The method used in [30] is supervised and requires labeled data. Moreover, even the similarity of the attribute "type" is learned through a training set. Regarding [4, 16, 21], the main contribution of these works relies on designing a spatial entity matching tool rather than an integration algorithm. The authors of [21] provide a general matching technique for spatial entity matching. Spatial entities within a radius are compared with each other, and the value of the radius is fixed depending on the type of spatial entity. For example, the radius is $50 \mathrm{~m}$ for restaurants and hotels, but $500 \mathrm{~m}$ for parks. All attributes (except coordinates) are compared using the Levenshtein similarity. Since the name, the geodata and the type of the entity are always present, they carry two-thirds of the weight in the scoring function whereas the weights of the website, the address and the phone number are tuned to one-third. The prototype of the spatial entity matching in [4] relies on a technique that arbitrarily uses an average of the similarity scores of all textual attributes without providing a discussing on this choice. Similarly to $[4,16]$, the main contribution of the work in [21] is designing a tool for spatial entity integration. The underlying algorithm considers spatial entities that are $5 \mathrm{~m}$ apart from each other and compares the name of the entities syntactically and the metadata related to an entity semantically. Finally, the decision is taken using the belief theory [27]. The works in $[4,16,21]$ lack an evaluation of the algorithms.

Summary. On the one hand, the general entity resolution approaches propose interesting solutions, but they do not consider the spatial character of a spatial entity. They do not deal with geographic coordinates and are designed to match entities that represent individuals (profiles in social networks, authors and publications, medical records, genealogical connections, etc.) or even linking species in nature. The proposed solutions for the former [entity resolution in individuals], either supervised or based on an experimental threshold, are learned on human entity datasets. One can not merely assume the resemblance of behaviors in a human entity dataset to a spatial entity one. The solutions in the latter [species in nature] are based on domain-specific algorithms that have little to no applicability in other fields. On the other hand, there is little specific work in spatial entities [4, 16, 21], mostly focusing on a tool for spatial data integration rather than on the algorithm. In all these works, the scoring function is chosen arbitrarily and no evaluation provided.

\section{SPATIAL ENTITY LINKAGE}

In this section, we introduce definitions, the problem of spatial entity linkage, and our proposed solution.

\subsection{Problem Definition}

The basic concept used in this work is a spatial entity, used for locations, places, businesses, etc. Spatial entities originate from 
location-based sources such as directories with location information (yellow pages, Google Places, etc.) and location-based social networks (Foursquare, Gowalla, etc.).

Definition 3.1. A spatial entity $s$ is an entity identified uniquely within a source $I$, located in a geographical point $p$ and accompanied by a set of attributes $A=\left\{a_{i}\right\}$.

The attributes connected to an $s$ can be categorized as:

- Spatial: the point where the entity is located, expressed in longitude and latitude.

- Textual: attributes that are in the form of text such as name, address, website, description, etc.

- Semantic: attributes in the form of text that enrich the semantics behind a spatial entity. Examples of this type are categories, keywords, metadata, etc.

- Date, time or number: other details about a spatial entity such as phone, opening hours, date of foundation, etc.

An example of a spatial entity originating from Yelp can be a place named "Star Pizza" in the point (56.716 10.114), with the keywords "pizza, fast food", and with address "Storegade 31". The same spatial entity can be found again in Yelp or other sources, sometimes having the same attributes, more, less or even attributes with contradictory values. Thus, there is a need for an approach that can unify information within and across different sources in an intelligent manner.

Problem definition: Given a set of spatial entities $S$ originating from multiple sources, the spatial entity linkage problem aims to find those pairs of spatial entities $\left\langle s_{i}, s_{j}\right\rangle$ that refer to the same physical spatial entity.

In the following section, we introduce QuadSky, our solution to the spatial entity linkage problem.

\subsection{QuadSky Approach}

We propose QuadSky, a solution based on a quadtree data partitioning and skyline exploration. The overall approach is detailed in Fig. 1. QuadSky consists of three main parts: spatial blocking (QuadFlex), pairwise comparisons and labelling the pairs (SkyEx). S contains all spatial entities from all sources I. We propose QuadFlex, a quadtree-based solution that can perform the spatial blocking by respecting the distance between spatial entities and the density of the area. The output of QuadFlex is a list of leaves with spatial entities located nearby. Within the leaves, we perform the pairwise comparisons of the attributes. After this second phase, we obtain a list of pairs of spatial entities and their similarities of attributes. In order to decide which pairs dictate a match and which not, we propose a novel technique, referred to as $S k y E x$ that explores $k$ skylines (concepts detailed in Section 3.5) of the pairs. SkyEx separates the pairs that refer to the same physical spatial entity (the positives class) from the rest (the negative class). In the following sections, we detail each of the phases of QuadSky.

\subsection{Spatial Blocking}

Performing all possible comparisons between pairs of spatial entities is time-consuming. Since spatial proximity is a strong indicator in finding a match, the first step is to group nearby spatial entities in blocks. Several generic blocking techniques have been discussed

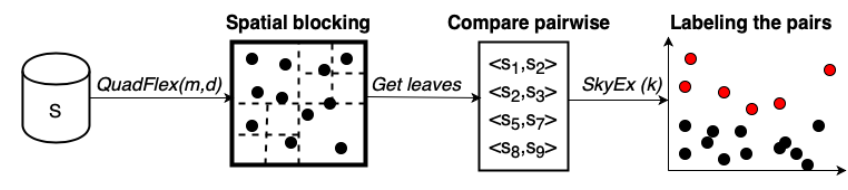

Figure 1: QuadSky approach

in [23, 24], including methods based only on one attribute or several ones, block purging (discards the blocks that are over the size limit), etc. However, these techniques are mostly based on textual attributes and not applicable to spatial blocking. We propose a quadtree-based solution (QuadFlex) that uses a tree data structure but also preserves the spatial proximity of spatial entities. Our contribution is twofold; we use quadtrees (meant for spatial indexing) for spatial blocking, and we modify the recursive procedure of the quadtrees to accommodate more points that are nearby instead of splitting them arbitrarily in different children.

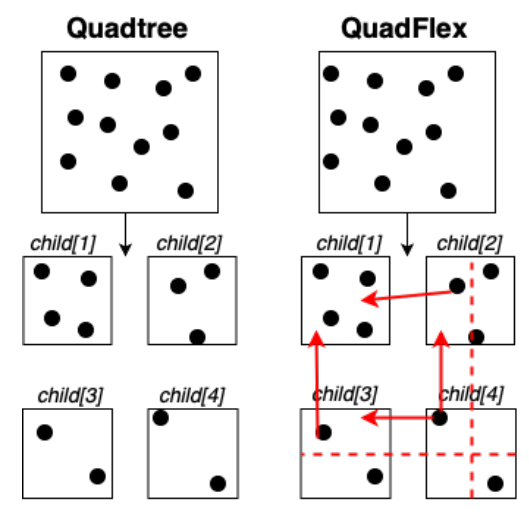

Figure 2: QuadFlex versus quadtree

A quadtree is a tree whose nodes are always recursively split into four children when the capacity is filled [28]. Quadtrees of $n$ points and $d$ depth can be constructed in $O((d+1) n)$ time. After the quadtree is constructed, the points that fall in the same leaf are nearby spatially. Hence, these leaves are good candidates to be spatial blocks. There are several issues with the existing quadtree algorithm. First, a quadtree needs a capacity (number of points) as a parameter. The capacity is not a meaningful parameter for spatial blocking, while the density of the area is a better candidate. For example, if the area is too dense (e.g., city center), even though the capacity is not reached, a further split would be more beneficial. Spatial entities in the city center tend to be nearby, but they are more unlikely to be the same. On the contrary, two points in the countryside (e.g., a farm) might be farther apart, but they still might be the same entity. Second, a quadtree does not limit the distance between points. Even though two points might be in an area that respects the density, if they are quite distant from each other, it is not necessary to compare them. The maximal distance between two points in a child is the diagonal of the area (all quadtree children are rectangular). We used $m$, the diagonal of an area, as a parameter that controls the distance of points rather than comparing all distances. Finally, a quadtree splits into four children, and sometimes nearby 
points might fall into different leaves. Hence, we might miss good pairs. We modify the procedure of the assignment of the points into a child by allowing more than one assignment.

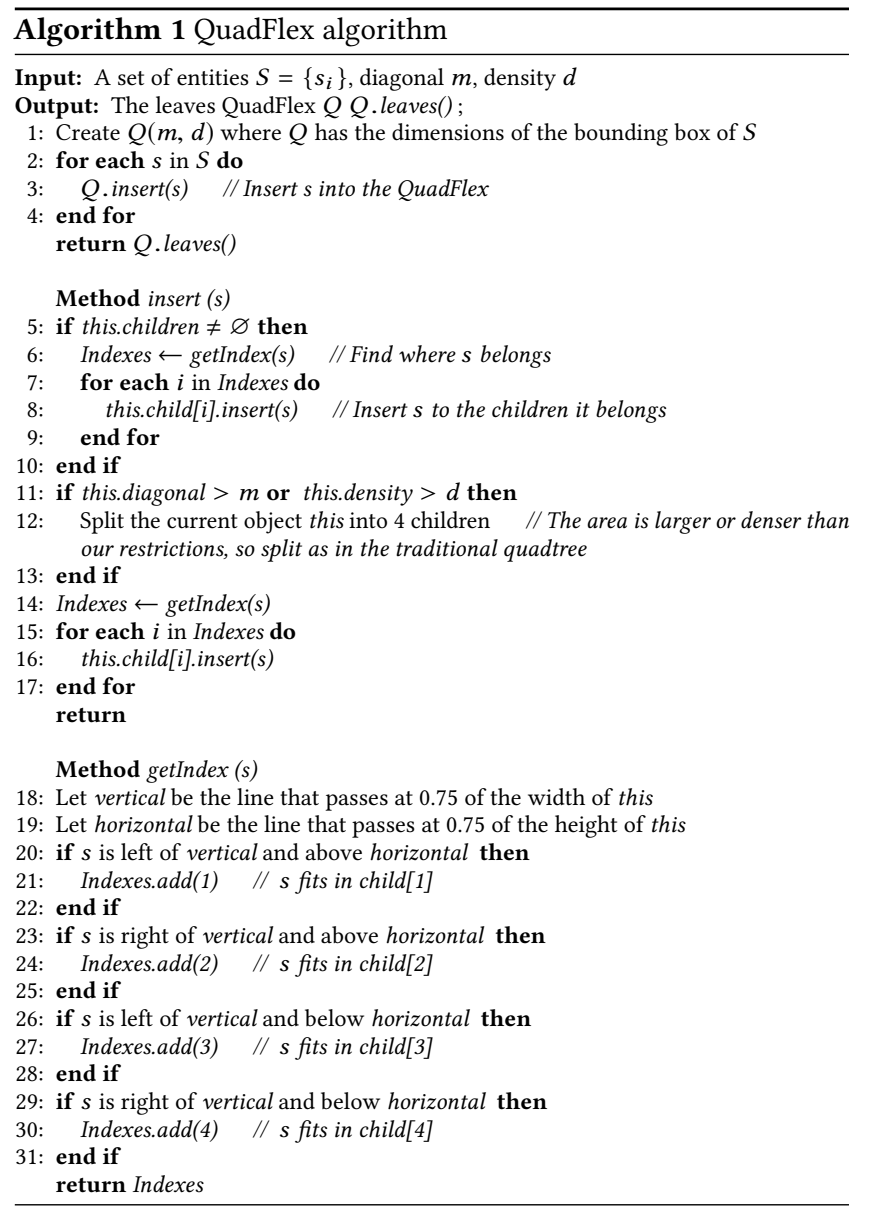

Fig. 2 shows the modifications that we do to the construction of the traditional quadtree for our version QuadFlex. The traditional quadtree divides the area of each parent into four smaller areas, the children. A point belongs only to one child. In our modification, the area will split similarly to the quadtree, but when we assign a point to a child, we will consider including points that fall near the border also in the current child. For example, in Fig. 2, the red dashed line shows the area that will be considered to include points in the neighbors. We will add two points in child[0], one coming from child[1] and one from child[3]. Similarly, child[1] and child[3] will receive a point from child[4].

Algorithm 1 details the procedure for retrieving the spatial blocks with QuadFlex. The algorithm creates the root of the QuadFlex tree with the bounding box of the data and parameters $m$ and $d$ (line 1). Then, it inserts each spatial entity into the QuadFlex (line 4) and finally returns its leaves. The methods insert(s) and getIndex(s) are self calls on the QuadFlex object (this). The insertion procedure is similar to the traditional quadtree except that the constraint is not the capacity but the diagonal of the area $m$ (maximal distance between points) and the density of the area $d$. Hence, if the diagonal of the QuadFlex is more than the distance $m$ or the density is larger than our defined value $d$ (line 12), the QuadFlex, similarly to a quadtree, will split into four children. However, in contrast to the traditional quadtree, a spatial entity might belong to more than one child. The method getIndex(s) gets the list of indexes of the children where the new point will be assigned. Even though $Q$ splits into 4 children, the lines vertical and horizontal (corresponding to the red dashed lines in Fig. 1) allow a logical overlap of the areas and thus, neighboring spatial entities will not be separated.

\subsection{Pairwise Comparisons}

After the spatial blocking, we perform a pairwise comparison of spatial entities that fall in the same leaf. Next, we describe the metrics for different types of attributes.

Textual Similarity. We measure the textual similarity of spatial entities using the edit distance between the words. The Levenshtein distance [18] between string $s_{1}$ and string $s_{2} d\left(s_{1}, s_{2}\right)$ is the number of edits (insertion, deletion, change of characters) needed to convert string $s_{1}$ to string $s_{2}$. We define the similarity as:

$$
\operatorname{TextSim}\left(s_{1}, s_{2}\right)=\left(1-\frac{d\left(s_{1}, s_{2}\right)}{\max \left(\left|s_{1}\right|,\left|s_{2}\right|\right)}\right)
$$

Example 3.1. Let us consider "Skippers Grill" and "Skippers Grillbar". The Levenshtein distance to convert "Skippers Grill" to "Skippers Grillbar" is 3 ( 3 insertions). The lengths of the first and the second string are 14 and 17 respectively. So, TextSim("Skippers Grill", "Skippers Grillbar") $=1-(3 / \max (14,17)=0.8235$.

Note here that not all textual attributes can be handled similarly. String similarity metrics are usually appropriate for attributes like names, usernames, etc. Some other textual attributes require other metrics that need to be customized. In this paper, we consider address as a specific textual attribute. The similarity between two addresses cannot be measured with Levenshtein, Jaccard, Cosine, etc. since a small change in the address might be a giant gap in the spatial distance between the entities. For example, "Jyllandsgade 15 9480 Løkken" and "Jyllandsgade 759480 Løkken" have a distance of 1 and Levenshtein similarity of 0.963 , but they are 650 meters apart. However, "Jyllandsgade 159480 Løkken" and "Jyllandsgade 159480 Løkken Denmark" have a distance of 8 and Levenshtein similarity of 0.772 , but they are the same building. In $[4,16]$ the address is considered as another textual attribute without using its real semantics. In our case, we perform some data cleaning (removing commas, punctuation marks, lowercase, etc.), and then we search for equality $\left(s_{1}=s_{2}\right)$ or inclusion $\left(s_{1} \subset s_{2}\right.$ or $\left.s_{2} \subset s_{1}\right)$. We assign a similarity of 1 in the case of equality and 0.9 in the case of inclusion. Otherwise, the strings are not considered the same.

Semantic Similarity. The similarity of fields like categories, keywords or metadata cannot be compared only syntactically. Sometimes, several synonyms are used to express the same idea. Thus, we need to find a similarity than considers the synonyms as well. We use Wordnet [11] for detecting the type of relationship between two words and Wu\&Palmer similarity measure (wup) [34], which calculates how related two words are by taking into account the depths of the two synsets (sets of synonyms) in WordNet, along with the depth of the Least Common Subsumer (the most specific 
concept that is an ancestor of both words). The semantic similarity between two spatial entities is the maximal similarity between their list of categories,keywords or metadata. The semantic similarity of the spatial entities $s_{1}$ and $s_{2}$ is:

$$
\operatorname{SemSim}(s 1, s 2)=\max \left\{w u p\left(c_{i}, c_{j}\right)\right\}
$$

where $c_{i} \in C_{1}$ and $c_{j} \in C_{2}$ and $C_{1}$ is the set of keywords of $s_{1}$ and $C_{2}$ is the set of keywords $s_{2}$.

Example 3.2. Let us take an example of two spatial entities $s_{1}$ and $s_{2}$ and their corresponding semantic information expressed as keywords $C_{1}=\{$ "restaurant", "italian" $\}$ and $C_{1}=\{$ "food", "pizza" $\}$. The similarity between each pair is wup("restaurant", "food") = 0.4 , wup("italian", "food") $=0.4286$, wup("restaurant", "pizza") = 0.3333 and wup("italian", "pizza") $=0.3529$. Finally, the semantic similarity of $s_{1}$ and $s_{2}$ is $\operatorname{SemSim}(s 1, s 2)=\max \{0.4,0.4286,0.3333$, $0.3529\}=0.4286$.

Date, time or numeric similarity. The similarity between two fields expressed as numbers, dates, times or intervals is simply a boolean decision (true or false). For example, if the phone numbers change with only one digit, they are still different. Even though the similarity of these fields relies only on an equality check, most of the effort is put in data preparation. For example, before the comparison, the different phone formats should be identified and cleaned from prefixes. Other data formats like intervals (opening hours) might require temporal queries for similarity, inclusion, and intersection of the intervals. In this paper, we do not use these attributes for measuring the similarity between spatial entities, but for constructing the ground truth.

Summary. Spatial entities are characterized by different spatial attributes that differ in formats but also in the semantics. Textual attributes are the most studied in the literature, and several similarity metrics have already been proposed. We use Levenshtein while comparing the names of spatial entities, and customized comparisons for the address. Thus, we check for equality or inclusion to detect similar addresses. Metadata, keywords, categories, etc. are attributes that carry semantics. Since textual similarity cannot measure the similarity between synonyms, we rely on Wordnet to detect similar keywords attached to the spatial entities. Finally, other formats such as numbers, intervals, timestamps, etc. can be checked for equality, but also some background knowledge might be needed to detect matches.

\subsection{Labeling the Pairs}

After the pairwise comparison, the pairs are represented as points in a space with $n$ dimensions, where each dimension is an attribute. A pair has $n$ similarity values, one for each attribute. We denote as $\delta_{a}$ the similarity of two spatial entities for attribute $a$. For example, a pair $\left\langle s_{1}, s_{2}\right\rangle$ is represented as $\left(\delta_{a_{1}}, \delta_{a_{2}} \ldots \delta_{a_{n}}\right)$. The problem that we need to solve is which $\left\langle s_{i}, s_{j}\right\rangle$ pairs indicate a strong similarity and should be considered for a match. This is an old problem in the entity linkage community. A classifier $[9,13,17]$ can learn the behavior of the matches and detect the positive class. However, it is difficult to obtain labeled data, especially across different sources. Moreover, there is always the risk of overfitting when training a classifier on a sample. Some works assign weights on the similarity scores and test different combinations [17, 22, 26]. The problem that arises with these methods is that finding the best combination might require extensive experiments and might overfit the data.

We propose a more relaxed technique that uses Pareto optimality [6] for filtering the positive class. A solution $(x, y)$ is Pareto optimal when no other solution can increase $x$ without decreasing $y$. The points in the same Pareto frontier or skyline have the same utility. Widely used in economics and multi-objective problems, Pareto optimality is free of weights and similarity score functions. In the context of entity resolution, the skylines provide a selection of points that are better than others, but without quantifying how much better. The pairs that refer to the same physical spatial entity (the positive class) are expected to have high values of $\delta$. In general, the positive class is the minority and is spread all over the dataset, resembling outliers. This means that the first Pareto optimal frontier might contain only a couple of points. Thus, an exploration of several skylines ( $k$ levels) is needed. Under the assumption that the best values of $\delta$ belong to the pairs from the positive class, we label the $k$ skylines as the positive class and the rest as the negative. To the best of our knowledge, we are the first to propose a Pareto optimal solution for detecting matches for an entity linkage/resolution problem.

Definition 3.2. An attribute $a$ is positive discriminating if its similarity $\delta_{a}$ indicates a positive class rather than a negative.

An example of a positive discriminating attribute is the similarity of name. A higher name similarity is more likely to indicate a match than a non-match. For example, the name similarity for Mand \& Bil and Mand og Bil is 0.75, and for Solid and Sirculus ApS is 0.16. Hence, the former pair has a higher probability of being a match than the second. Examples of negative discriminating attributes are the edit distance between two names. If the distance between the names is high, then the pairs are less likely to be a match.

Definition 3.3. The utility of a positive discriminating attribute $a$ to the positive class, denoted as $u_{a}$, is a monotonically increasing function that quantifies the contribution of the similarity of attribute $\delta_{a}$ to indicate a match.

Each attribute contributes to the labeling problem. A higher similarity $\delta_{a}$ of $a$ has a higher utility than a lower value of $\delta_{a}$. Hence, if $\delta_{a}\left(\left\langle s_{1}, s_{2}\right\rangle\right)>\delta_{a}\left(\left\langle s_{3}, s_{4}\right\rangle\right)$, then $u_{a}\left(\left\langle s_{1}, s_{2}\right\rangle\right)>u_{a}\left(\left\langle s_{3}, s_{4}\right\rangle\right)$.

Definition 3.4. The utility of a pair denoted as $u\left(\left\langle s_{i}, s_{j}\right\rangle\right)$ is sum of the utilities of each of the attributes. $u\left(\left\langle s_{i}, s_{j}\right\rangle\right)=\sum_{i=1}^{n} u_{a_{i}}$.

Note that the utility of a pair is not the sum of the similarities of the attributes $\left(u\left(\left\langle s_{i}, s_{j}\right\rangle\right) \neq \sum_{i=1}^{n} \delta_{a_{i}}\right)$ but the sum of their utilities $\left(u\left(\left\langle s_{i}, s_{j}\right\rangle\right)=\sum_{i=1}^{n} u_{a_{i}}\right)$. Nevertheless, $u\left(\left\langle s_{i}, s_{j}\right\rangle\right)=\sum_{i=1}^{n} \delta_{a_{i}}=$ $\sum_{i=1}^{n} u_{a_{i}}$ is a specific case.

Definition 3.5. A skyline of level $k$ denoted as Skyline $(k)$ is the collection of pairs $\left\langle s_{i}, s_{j}\right\rangle$ of equal utility such that $u_{S k y l i n e(k)}>$ $u_{\text {Skyline }(k-1)}$.

Obviously, Skyline(1) is the Pareto optimal frontier with the best values of $\delta_{a}$. In order to continue with Skyline(2), the points of Skyline(1) are removed, and the frontier is calculated again. Every time we explore level $k$, the values in $\operatorname{Skyline}(k)$ are the ones with the highest utility. This means that there is no other point in a lower 
level that can bring a higher utility to the positive class. This procedure continues for $k$ steps. Algorithm 2 formalizes our proposed procedure Skyline Explore ( $S k y E x$ ) for labelling the pairs. The input is the set of pairs $P$ produced from the QuadFlex blocking technique and the number of skyline levels $k$ that we will explore. We find the points with the best combinations of $\delta$ that dominate the rest of the points and consequently, have a higher utility (line 3). Then, we put these points in $P^{+}$and remove them from $P$ (line 5). After all $k$ levels are computed, we return the positive set of pairs $P^{+}$and the negative $P^{-}$

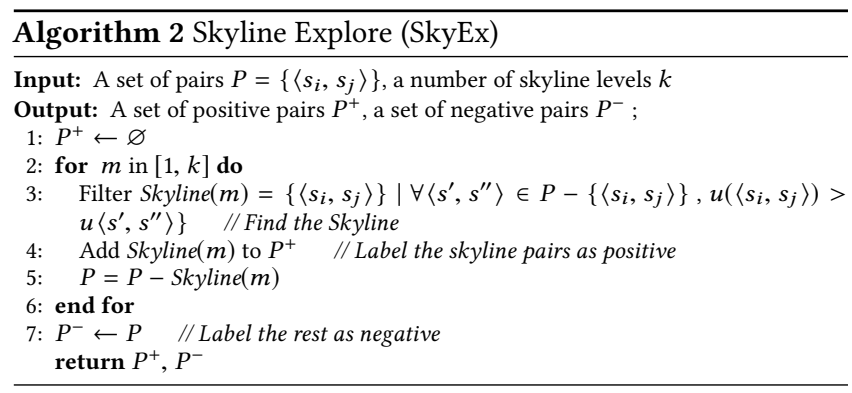

In contrast to techniques that use a similarity score function, $S k y E x$ abstracts the concept of utility. Thus, no weights or similarity function is needed. Given that the points with a high utility are generally scattered, $S k y E x$ can detect the positive class better than a clustering technique, which would fail in clustering together the positive class. Moreover, the flexibility of $S k y E x$ makes it applicable to all problems where the expert knowledge on the contribution of the attributes is missing. Finally, $S k y E x$ does not learn any behavior, so there is no risk of overfitting.

\section{EXPERIMENTS}

\subsection{Dataset Description}

The spatial entities that will be used in these experiments originate from four sources, namely Google Places (GP), Foursquare (FSQ), Yelp, and Krak. Krak (www.krak.dk) is a location-based source that offers information about companies, enterprises, etc. in Denmark and is also part of Eniro Danmark A / S., which publishes The Yellow Pages. The data is obtained by using the available APIs and the algorithm detailed in [14]. The distribution of the spatial entities can be observed in Fig. 3. The dataset consists of 75,541 spatial entities where $51.50 \%$ comes from GP, $46.22 \%$ from Krak, $0.03 \%$ from FSQ, and $2.23 \%$ from Yelp. All the sources internally might contain possible links, so we need to compare entities within and outside the sources.

\subsection{QuadFlex Performance}

In this section, we compare the performance of our proposed blocking technique to the traditional quadtree and Fixed Radius Nearest Neighbors algorithm [3] (FNN). FNN finds the neighbors that fall within a fixed radius from each point. QuadFlex and the quadtree algorithm are implemented in Java, while FNN is run on a Postgres database (https://www.postgresql.org) using spatial indexes; more specifically, two spatial indexes: GiST (https://www.postgresql.org/ docs/current/gist.html) (optimized C implementation of B-trees and

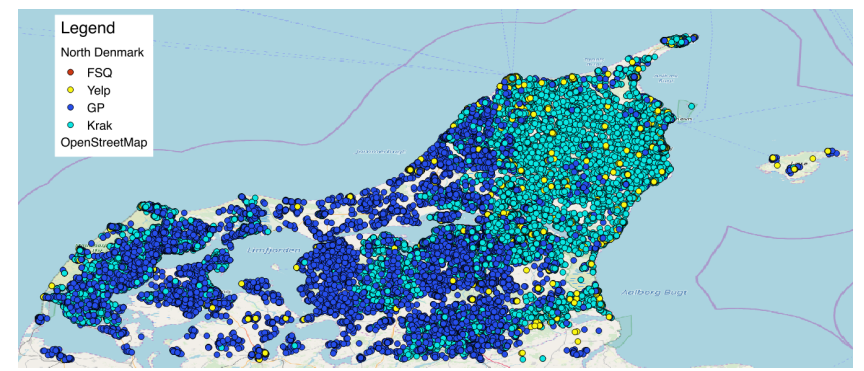

Figure 3: North Denmark dataset

R-trees) and SP-GiST (https://www.postgresql.org/docs/current/ spgist.html) (optimized C implementation of quadtrees and k-d trees). Our original dataset contains 75,541 entities in the whole North Denmark region (around 16 towns, 7,933 $\mathrm{km}^{2}$ ), so the density is not high. A high data density means more neighbors and consequently, more pairs to compare. In order to test our QuadFlex on different data densities, we simulate up to $1,000,000$ random points from Aalborg $\left(139 \mathrm{~km}^{2}\right)$.

Fig. 6 shows the comparison of quadtree, QuadFlex and FNN in terms of execution time (Fig. 6a) and number of comparisons (Fig. 6b). The FNN versions with data are computed on the database, and then the pairs are loaded back to the java implementation. The quadtree has the lowest execution time, followed by QuadFlex. FNN SP-GiST is comparable and sometimes even better than QuadFlex for small datasets. However, when the size of the dataset increases, QuadFlex manages to maintain an execution time that is eight times less than FNN GiST and 3 times less than FNN SP-GiST. FNN with SP-GiST index outperforms FNN GiST for all dataset sizes. As for the number of comparisons, QuadFlex enumerates 12 times more comparisons than quadtree, so our technique for not missing nearby pairs turned out effective. Moreover, QuadFlex contains almost all (99.99\%) comparisons of FNN, compared to quadtree that contains only $10 \%$ of FNN. Furthermore, given that the scalability of QuadFlex is better than FNN, and QuadFlex is independent of the database implementations, the loss of around $0.01 \%$ of comparisons is insignificant. Moreover, this difference in comparisons can simply be explained by the fact that QuadFlex uses a rectangular area with diagonal of $m$ meters, whereas FNN uses a circle with radius $\frac{m}{2}$. In the case of a square with diagonal $m$, the surface will be $\frac{m^{2}}{2}$, but for the circle with the diameter $m$ the surface is $\pi \frac{m 2}{4}$. So, the surface of the circle is $\frac{\pi}{2}$ times more.

\subsection{SkyEx results}

In this section, we evaluate the results of our proposed $S k y E x$. In the context of our problem, we define true positives $T P$ as pairs that refer to the same physical entity and correctly labeled as positives, true negatives $T N$ as pairs referring to different physical entities and correctly labeled as negatives, false positive $F P$ as pairs that do not refer to the same physical entities but wrongly labeled as positives, and $F N$ as pairs that refer to the same physical entity but wrongly labeled as negatives. We measure precision $=\frac{T P}{T P+F P}$, recall $=\frac{T P}{T P+F N}$ and $F$-measure $(F 1)=2 \frac{\text { precision } * \text { recall }}{\text { precision }+ \text { recall }}$. 


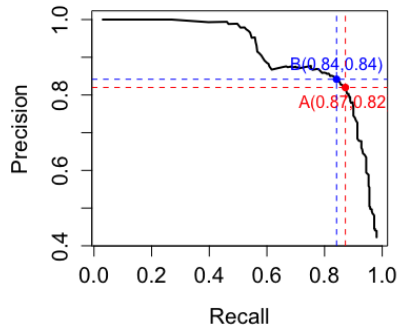

(a) Precision and recall

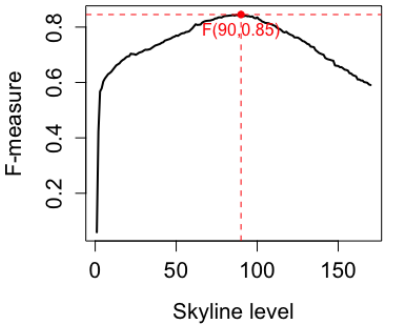

(b) F-measure
Figure 4: SkyEx performance on $D_{\text {sample }}$

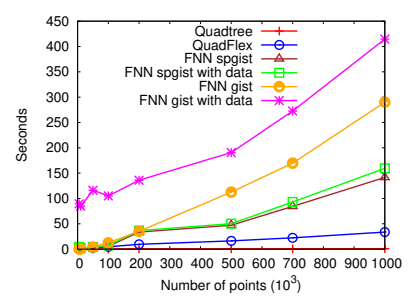

(a) Execution time

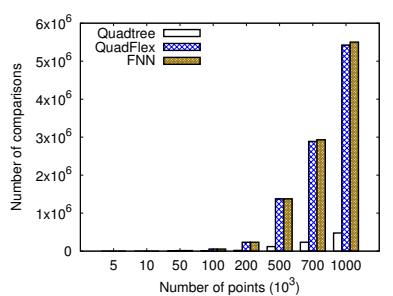

(b) Number of comparisons

Figure 6: Comparing quadtree, QuadFlex and FNN

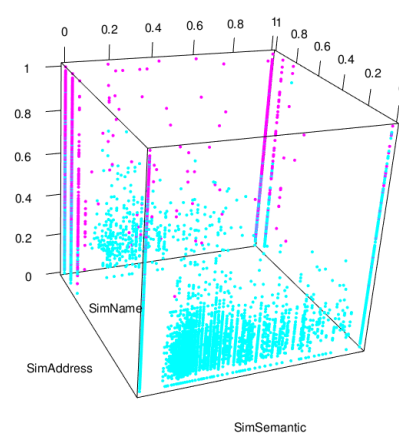

(a) Actual classes

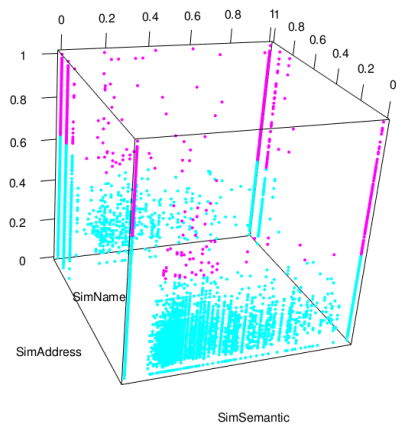

(b) SkyEx classes
Figure 7: Positive (in pink) versus negative (in sky blue) classes for actual (a) and SkyEx (b) results

We ran QuadFlex with $100 \mathrm{~m}$ and no density restriction, and we obtained 777,452 pairs. Having the same website or phone is a strong indicator of a match, so we use these attributes to infer the label. We refer to this labeling as automatic labeling. However, cases with different phone number or website but still the same entity, or same phone number but different entity might occur. For example, an entrepreneur who owns a fishing company and also a restaurant might use the same phone for both. Another example is the case of two different phones for the same entity on different sources. For this reason, we manually checked the labels of a sample of 1,500 pairs of entities, sometimes checking them even on maps and on the

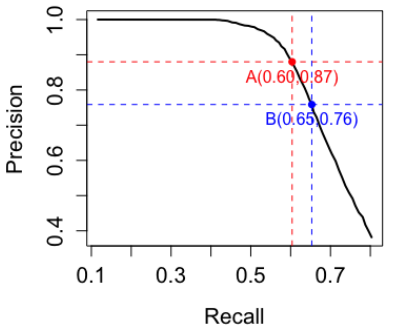

(a) Precision and recall

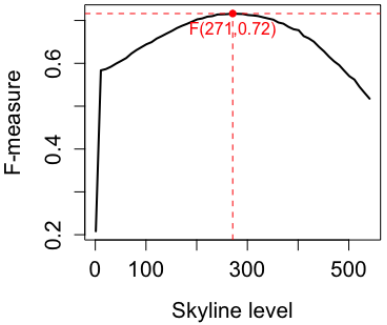

(b) F-measure
Figure 5: SkyEx performance on $D_{\text {full }}$

original sources. We will refer to the sample of manually checked pairs as $D_{\text {sample }}$ and to the full dataset as $D_{\text {full }}$. Checking the labels manually on the full dataset of 777,452 pairs is unfeasible. Hence, we checked around 10,000 of the pairs, and for the rest, we rely on automatic labeling.

The results of SkyEx on $D_{\text {sample }}$ and $D_{\text {full }}$ are presented in Figs. 4 and 5. The curves in Figs. 4a and 5a shows the evolution of precision ( $y$-axis) and recall ( $x$-axis) while we move from one skyline to the next. As expected, the more we explore, the more likely it is to retrieve more true positives and thus, improve the recall. However, the more we explore and label pairs as positives, the more likely it is to increase the number of false positives, so the precision degrades. The algorithm explores several trade-offs, where points $A$ and $B$ are among the best. Point $A$ with 0.87 precision and 0.82 recall in Fig. 4a is the same best point in terms of F-measure as well. Fig. $4 \mathrm{~b}$ shows the levels of the skyline, and the value of $F$-measure achieved. The highest value is 0.85 that corresponds to $k=90$. Point $B$ is also a good candidate with 0.84 precision and recall.

The evaluation on the full dataset yields lower values compared to the sample, which might be a simple consequence of automatic labeling. Even though the labels are not all checked, precision and recall in $D_{\text {full }}$ yield satisfactory values. Two of the best combinations are points $A$ and $B$, where $A$ is the combination also with the highest F-measure of 0.72 and 271 skyline levels Fig. 5b. $A$ offers 0.6 recall and 0.87 precision while $B$ offers a higher recall of 0.65 but a lower precision of 0.76 . To have an idea of the real classes in $D_{\text {full }}$ and the skyline, we plotted their distribution in Fig. 7. Fig. 7a shows the actual positive classes in pink and the negative ones in sky blue. It is noticeable that the positive class pairs are allocated in the highest values of the dimensions. SkyEx with 271 levels (Fig. 7b) is able to capture this behaviour and achieve 0.6 recall and 0.87 precision. Despite the differences between both plots, SkyEx shows promising results in separating the positive class from the negative one.

\subsection{Experimenting with Different QuadFlex Parameters}

So far, we used QuadFlex blocking technique with 100 meters and no density restriction. In this section, we will evaluate our approach QuadSky for different blocking parameters. 


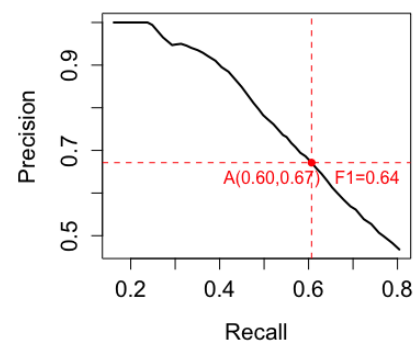

(a) $\mathrm{m}=1$

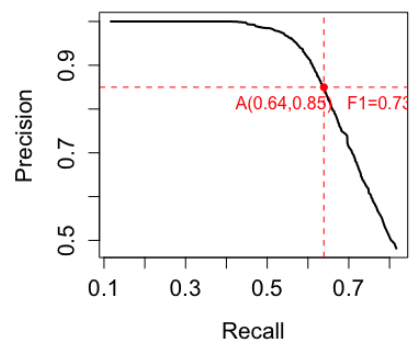

(d) $\mathrm{m}=60$

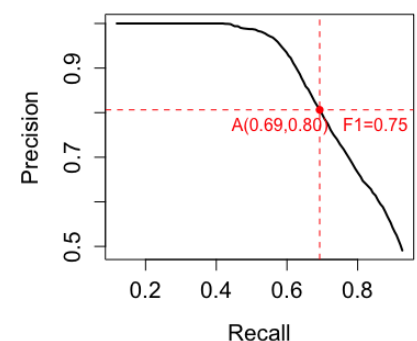

(b) $\mathbf{m}=20$

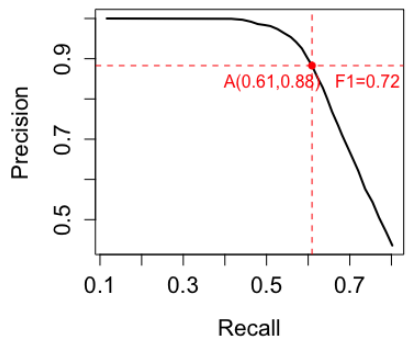

(e) $\mathbf{m}=\mathbf{8 0}$

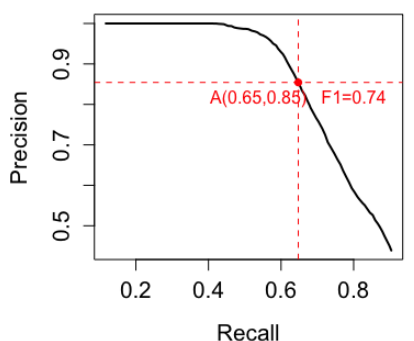

(c) $\mathrm{m}=\mathbf{4 0}$

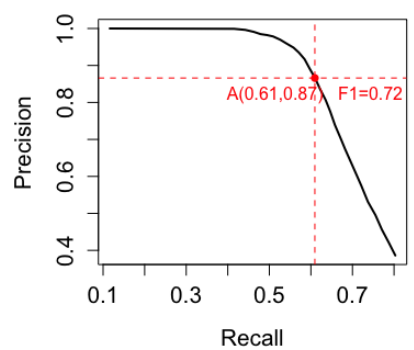

(f) $\mathrm{m}=\mathbf{1 0 0}$

Figure 8: Performance of SkyEx for different m, no density limit

Changing $\boldsymbol{m}$, no density limit. In this experiment, we test different values of $m$ used in QuadFlexfor creating spatial blocks. We test $m$ values of $1,20,40,60,80$, and 100 meters. The size of the dataset for each of them is presented in Table 1 . The spatially close points are likely to be a match. Hence, the percentage of the positive class is generally higher for smaller values of $m$. An interesting case is $m=1$, where the percentage of the positive class is lower than $m=20$. One would expect that points that are 1 meter apart would unquestionably be a match. However, this is not always the case. Shopping malls, buildings that host several companies, etc. are characterized by the same coordinates but not necessarily the same spatial entities.

\begin{tabular}{lllllll}
\hline Meters & 1 & 20 & 40 & 60 & 80 & 100 \\
\hline Total & 41053 & 118437 & 226331 & 372553 & 557421 & 777452 \\
\% of pos & $17.11 \%$ & $19.88 \%$ & $11.28 \%$ & $7.06 \%$ & $4.82 \%$ & $3.49 \%$ \\
\hline
\end{tabular}

Table 1: Dataset characteristics for different $\mathbf{m}$

The results for different values of $m$ are presented in Fig. 8. The point $A$ is the value with the highest $F$-measure $(F 1)$. For all cases, the recall is higher than 0.6 . The precision is higher than 0.8 for all values of $m$, except $m=1$, where the precision is 0.67 . For $m=1$, the positive and negative class are mixed, thus $S k y E x$ loses a bit in precision. This is also an argument against the works that merge arbitrarily points that are $5 \mathrm{~m}$ apart. Spatial proximity is not a definitive indicator of a match.

Changing $\boldsymbol{d}, \boldsymbol{m} \leq \mathbf{1 0 0}$. The density is another parameter of QuadFlex that helps in creating smaller blocks in dense areas and larger ones in sparse areas. In this experiment, we test different values of density $d$ and its effect on the results. The size of the dataset and the percentage of the classes in Table 2. When the density is smaller, we force QuadFlex to split further and create smaller blocks. Thus, the number of pairs reduces. Note that, on the contrary, the percentage of positives increases. Indeed, further splits allow us to create better blocks containing a higher percentage of positives. However, when the density increases above $\frac{30 \mathrm{~s}}{1000 \mathrm{~m}^{2}}$, fewer and fewer blocks are split further, so the dataset size and the percentage of the positives do not vary significantly.

\begin{tabular}{lllllll}
\hline Density & $\frac{10 s}{1000 m^{2}}$ & $\frac{20 s}{1000 m^{2}}$ & $\frac{30 s}{1000 m^{2}}$ & $\frac{40 s}{1000 m^{2}}$ & $\frac{50 s}{1000 m^{2}}$ & $\frac{60 s}{1000 m^{2}}$ \\
\hline Total & 290653 & 590583 & 711423 & 754195 & 770987 & 776664 \\
\% pos & $8.61 \%$ & $4.57 \%$ & $3.81 \%$ & $3.59 \%$ & $3.51 \%$ & $3.49 \%$ \\
\hline
\end{tabular}

Table 2: Dataset characteristics for different $\mathrm{d}$

The results after running $S k y E x$ are presented in Fig. 9. In all the cases, the recall stays above 0.61 and the precision above 0.87 . A slightly better precision (0.88) and recall (0.63) is achieved in the case of a density of $\frac{10 \mathrm{~s}}{1000 \mathrm{~m}^{2}}$ (the lowest parameter). From both experiments with QuadFlex parameters, we can conclude that $S k y E x$ adapts very well in finding the correct classes even when the size of blocks change and even when the percentage of positives over negatives varies.

\subsection{Comparison with Baselines}

Even though there are several papers in spatial data integration, the works of $[4,16,21]$ are the most similar to ours, as the rest of the related work considers only spatial objects, not spatial entities, 


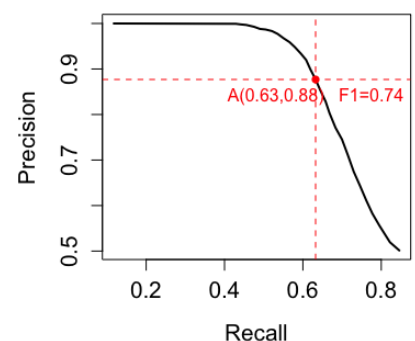

(a) $d=10 / 1000 \mathrm{~m}^{2}$

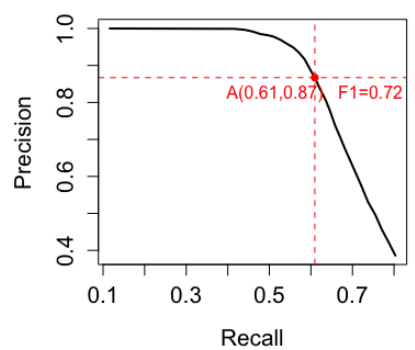

(d) $\mathrm{d}=40 / 1000 \mathrm{~m}^{2}$

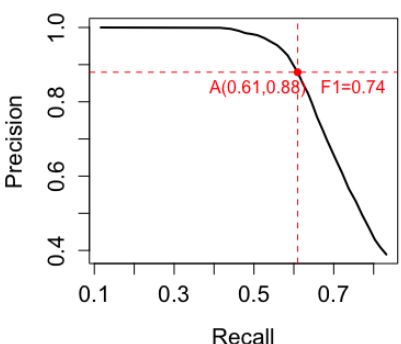

(b) $d=20 / 1000 \mathrm{~m}^{2}$

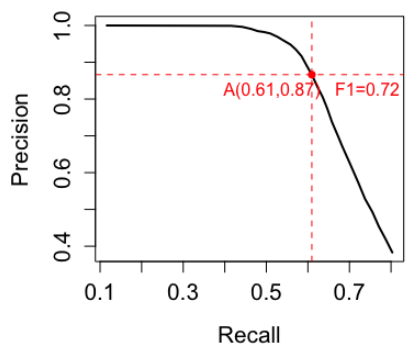

(e) $d=50 / 1000 \mathrm{~m}^{2}$

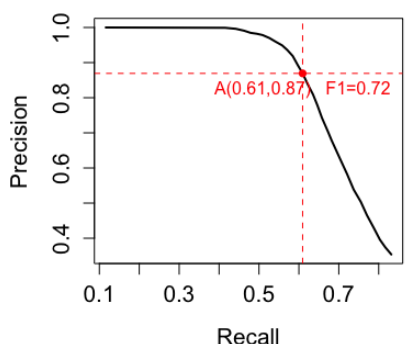

(c) $d=30 / 1000 \mathrm{~m}^{2}$

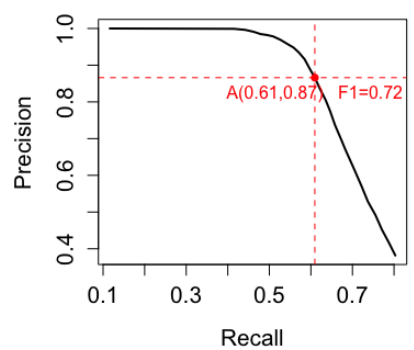

(f) $d=60 / 1000 \mathrm{~m}^{2}$

Figure 9: Performance of SkyEx for different $d, m=100$

or uses supervised learning techniques. We will compare QuadSky to the following baselines:

(1) Berjawi et al.[4] propose Euclidean distance for the geographic coordinates and Levenshtein similarity for all other attributes. The similarities are integrated into a global similarity computed as a simple sum of the attribute similarities. The attributes mentioned in the paper are the name and the phone. However, since the phone is part of our automatic labeling, it can not be used in the algorithm as well. The authors consider pairs with score $\geq 0.75$ as a match with high confidence. We compare to this technique using name + address + geographic coordinates (V1) and name + geographic coordinates (V2).

(2) Morana et al.[21] suggest filtering entities that share the same category or a token in the name. Then these entities are compared using the Euclidean distance for the coordinates, Levenshtein for the address and name, and Resnik similarity (Wordnet) for the category. Attributes like address, phone, etc. are considered secondary, so they are given $\frac{1}{3}$ of the weight in the similarity score function, while name, category and geographic proximity carry $\frac{2}{3}$ of the weight. The authors show top $k$ matches for each entity to the user to decide.

(3) Karam et al.[16] starts with filtering spatial entities that are $5 \mathrm{~m}$ apart. Then, the similarity of the name is measured with Levenshtein distance, the geographic similarity with Euclidean distance, and the keywords are compared semantically. In order to decide which pairs to match and which not, the similarities are fused using belief theory [27].

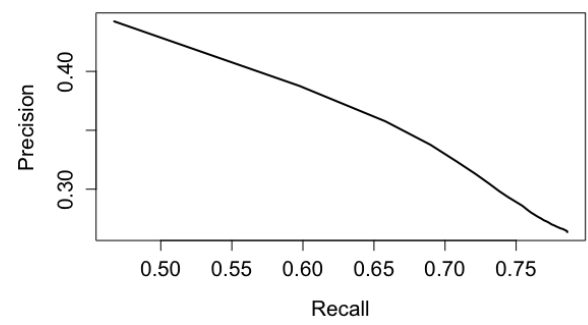

Figure 10: Performance of Morana et al.[21] on $D_{\text {full }}$

The results using $D_{\text {full }}$ and $D_{\text {sample }}$ are presented in Table 3. In general, all the methods performed better in the manually labeled dataset $D_{\text {sample }}$ due to the better quality of the labels. Berjawi et al.(V1)[4] has the highest precision of above 0.93 , but a poor recall of at most 0.27 for both datasets and thus, a low F-measure of at most 0.43 . It is actually expected, as Levenshtein similarity does not perform well with fields like address or phone, where a change in the digits makes a huge difference in the similarity of attributes. Berjawi et al.(V2)[4] yields reasonable results, the second best after QuadSky, with a precision of 0.73 in $D_{\text {full }}$ and 0.97 in $D_{\text {sample }}$, and a recall of 0.56 in $D_{\text {full }}$ and 0.60 in $D_{\text {sample. }}$. The F-measure is 0.63 in $D_{\text {full }}$ and 0.74 in $D_{\text {sample }}$.

To compare with Morana et al.[21], we tried all values $k$ from 1 to the maximal matches for a single point. We plotted all combinations of precision and recall for different values of $k$ in Fig. 10 for $D_{\text {full }}$. The highest value of F-measure corresponded to a precision of 0.39 and a recall of 0.60 . The behavior of Morana et al.[21] in $D_{\text {sample }}$ is similar; the best value of F-measure was achieved for $k=3$ and 
results are similar to those in $D_{\text {full }}$. The work of Karam et al.[16] achieves the highest recall of 0.73 but a very low value of precision of 0.23 for $D_{\text {full }}$. As a result, the F-measure is only 0.47 . However, in $D_{\text {sample }}$, the method performs better overall ( F-measure $=0.6$ ).

In comparison to all the baselines, QuadSky provides the best tradeoff between precision and recall, and thus, the highest F-measure in both datasets. In the case of $D_{\text {sample }}$, QuadSky achieves the best recall compared to all baselines. The highest precision values for both datasets is achieved by Berjawi et al.(V1)[4] but a very low recall and poor model performance overall. In fact, models that achieve extreme values (high precision-low recall or low precisionhigh recall) are not a viable solution because they are either too restrictive or too flexible, and their predictability is poor. Most of the current work base their scoring function on the assumption that the geographical proximity is essential, so besides spatial blocks, they include Euclidean distance in their similarity functions. QuadSky uses the spatial proximity for identifying candidates but not for making a decision. Berjawi et al. [4] (V2) assumes the same weights for all similarities, and the reported values of precision and recall are reasonable. However, the behaviors of the pairs can be of all types. QuadSky can capture these different behaviors better than a simple sum would.

\begin{tabular}{lllllll}
\hline & \multicolumn{3}{c}{$D_{\text {full }}$} & \multicolumn{3}{c}{$D_{\text {sample }}$} \\
\hline Approach & Precision & Recall & $\mathbf{F 1}$ & Precision & Recall & F1 \\
\hline Berjawi et al.(V1)[4] & $\mathbf{0 . 9 3}$ & 0.26 & 0.41 & $\mathbf{1 . 0 0}$ & 0.27 & 0.43 \\
Berjawi et al.(V2)[4] & 0.73 & 0.56 & 0.63 & 0.97 & 0.60 & 0.74 \\
\hline Morana et al.[21] & 0.39 & 0.60 & 0.47 & 0.33 & 0.60 & 0.43 \\
\hline Karam et al.[16] & 0.23 & $\mathbf{0 . 7 3}$ & 0.35 & 0.54 & 0.68 & 0.60 \\
\hline QuadSky & 0.87 & 0.60 & $\mathbf{0 . 7 2}$ & 0.87 & $\mathbf{0 . 8 2}$ & $\mathbf{0 . 8 5}$ \\
\hline
\end{tabular}

Table 3: Comparison with the baselines

\section{CONCLUSIONS AND FUTURE WORK}

Location-based sources provide rich information about spatial entities in terms of details and semantics. However, identifying which pairs of spatial entities refer to the same physical entity across different sources is a challenging problem due to the lack of labeled data, data quality problems in the sources and the difficulty of coming up with a data independent scoring function. In this paper, we addressed the problem of spatial entity linkage across multiple location-based sources. We proposed QuadSky, an approach that consists of two novel algorithms QuadFlex and SkyEx. QuadFlex arranges the spatial entities into spatial blocks with a low execution time (4-8 times less than Fixed Radius Nearest Neighbors algorithm [3] (FNN)) and a high percentage of comparisons (99.99\% of FNN comparisons). SkyEx solves the data labeling problem using Pareto optimality and yields good results in terms of precision and recall. Moreover, due to its flexibility, $S k y E x$ better captures the behavior of pairs and outperforms the existing baselines in terms of F-measure. More specifically, $S k y E x$ achieves 0.84 precision and 0.84 recall on a manually labeled dataset and 0.87 precision and 0.6 recall on an automatically labeled dataset. In future work, we aim to study different blocking techniques that combine several attributes. Moreover, we plan to improve our proposed $S k y E x$ in order to automatically select the $k$ number of skylines based on the dataset characteristics.

\section{REFERENCES}

[1] R. Abdalla. 2016. Geospatial Data Integration. In GeoICT.

[2] S. Balley, C. Parent, and S. Spaccapietra. 2004. Modelling geographic data with multiple representations. IfGIS (2004).

[3] J. L. Bentley, D. F. Stanat, and E. H. Williams Jr. 1977. The complexity of finding fixed-radius near neighbors. Information processing letters (1977).

[4] B. Berjawi, E. Chesneau, F. Duchateau, F. Favetta, C. Cunty, M. Miquel, and R. Laurini. 2014. Representing Uncertainty in Visual Integration.. In DMS.

[5] D. G. Brizan and A. U. Tansel. 2006. A. survey of entity resolution and record linkage methodologies. Communications of the IIMA (2006).

[6] Yair Censor. 1977. Pareto optimality in multiobjective problems. Applied Mathematics and Optimization (1977).

[7] P. Christen, T. Churches, and M. Hegland. 2004. Febrl-a parallel open source data linkage system. In $P A K D D$.

[8] X. L. Dong and D. Srivastava. 2013. Big data integration. In ICDE.

[9] M. Edwards, S. M. Wattam, P. E. Rayson, and A. Rashid. 2016. Sampling labelled profile data for identity resolution. (2016).

[10] J. Efremova, B. Ranjbar-Sahraei, H. Rahmani, F. Oliehoek, T. Calders, K. Tuyls, and G. Weiss. 2015. Multi-source entity resolution for genealogical data. In Population reconstruction.

[11] C. Fellbaum. 2010. WordNet. In Theory and applications of ontology: computer applications.

[12] D. Firmani, B. Saha, and D. Srivastava. 2016. Online entity resolution using an oracle. PVLDB (2016)

[13] O. Goga, H. Lei, S. K. Parthasarathi, G. Friedland, R. Sommer, and R. Teixeira. 2013. Exploiting innocuous activity for correlating users across sites. In $W W W$.

[14] S. Isaj and T. B. Pedersen. 2019. Seed-Driven Geo-Social Data Extraction - Full Version. CoRR (2019).

[15] S. Isaj, N. Seghouani, and G. Quercini. 2019. Profile Reconciliation Through Dynamic Activities Across Social Networks. In CAiSE.

[16] R. Karam, F. Favetta, R. Kilany, and R. Laurini. 2010. Integration of similar location based services proposed by several providers. In NDT.

[17] H. Köpcke, A. Thor, and E. Rahm. 2010. Evaluation of entity resolution approaches on real-world match problems. VLDB (2010).

[18] V. Levenshtein. 1966. Binary codes capable of correcting deletions, insertions, and reversals. In Soviet physics doklady.

[19] M. Magnani and D. Montesi. 2010. A survey on uncertainty management in data integration. $7 D I Q(2010)$.

[20] R. Maskat, N. W. Paton, and S. M. Embury. 2016. Pay-as-you-go Configuration of Entity Resolution. In Transactions on Large-Scale Data-and Knowledge-Centered Systems XXIX.

[21] A. Morana, T. Morel, B. Berjawi, and F. Duchateau. 2014. Geobench: a geospatial integration tool for building a spatial entity matching benchmark. In ACM SIGSPATIAL

[22] A. Panchenko, D. Babaev, and S. Obiedkov. 2015. Large-Scale Parallel Matching of Social Network Profiles. In AIST.

[23] G. Papadakis, E. Ioannou, C. Niederée, T. Palpanas, and W. Nejdl. 2012. Beyond 100 million entities: large-scale blocking-based resolution for heterogeneous data. In WSDM.

[24] G. Papadakis, J. Svirsky, A. Gal, and T. Palpanas. 2016. Comparative analysis of approximate blocking techniques for entity resolution. PVLDB (2016).

[25] O. Peled, M. Fire, L. Rokach, and Y. Elovici. 2013. Entity matching in online social networks. In SocialCom.

[26] G. Quercini, N. Bennacer, M. Ghufran, and C. N. Jipmo. 2017. LIAISON: reconciLIAtion of Individuals Profiles Across SOcial Networks. In Advances in Knowledge Discovery and Management.

[27] A. O. Raimond and S. Mustière. 2008. Data matching-a matter of belief. In Headway in spatial data handling.

[28] Hanan Samet. 1984. The quadtree and related hierarchical data structures. CSUR (1984).

[29] M. Schäfers and U. Lipeck. 2014. SimMatching: adaptable road network matching for efficient and scalable spatial data integration. In ACM SIGSPATIAL PhD Workshop.

[30] V. Sehgal, L. Getoor, and P. D. Viechnicki. 2006. Entity resolution in geospatial data integration. In GIS.

[31] K. Shu, S. Wang, J. Tang, R. Zafarani, and H. Liu. 2017. User identity linkage across online social networks: A review. ACM SIGKDD Explorations Newsletter (2017).

[32] PG Tabarro, J. Pouliot, R. Fortier, and L. Losier. 2017. A WebGIS to Support GPR 3D Data Acquisition: A First Step for the Integration of Underground Utility Networks in 3D City Models. ISPRS (2017).

[33] V. Walter and D. Fritsch. 1999. Matching spatial data sets: a statistical approach. IJGIS (1999).

[34] Z. Wu and M. Palmer. 1994. Verbs semantics and lexical selection. In ACL.

[35] C. T. Yui, L. J. Liang, W. J. Soon, and W. Husain. 2011. A survey on data integration in bioinformatics. In ICIEIS. 